\title{
Chemsex, Identity Processes and Coping among Gay and Bisexual Men
}

\author{
Rusi Jaspal \\ Nottingham Trent University, UK
}

\begin{abstract}
Purpose

Chemsex constitutes a significant public health concern among gay and bisexual men (GBM). Using Identity Process Theory, this study focuses on GBM's motivations for engaging in chemsex and the functions that the practice performs for constructing a positive sense of self and for coping with psychological stress.
\end{abstract}

\section{Design/methodology/approach}

Sixteen GBM were interviewed and the qualitative data were analyzed using thematic analysis.

\section{Findings}

Individuals reported facing various stressors, such as homonegativity, rejection and HIV stigma, which were threatening for self-esteem and distinctiveness. There was habitual use of deflection (e.g., denial and self-concealment) for coping with these stressors. Chemsex enabled some interviewees to engage in more elaborate forms of deflection, such as transient depersonalization, compartmentalization and fantasy.

\section{Originality}

In contrast to the risk-focused analyses of chemsex, this study provides a novel identity-based approach to understanding GBM's motivations for engaging in chemsex and focuses on the functions that chemsex may perform for identity processes.

\section{Keywords}

Chemsex; gay and bisexual men; identity; coping; deflection

\section{Corresponding author}

Professor Rusi Jaspal, Department of Psychology, Nottingham Trent University, Nottingham NG1-4FQ, United Kingdom. E-mail: rusi.jaspal@cantab.net

\section{Citing this article}

Jaspal, R. (2021). Chemsex, identity processes and coping among gay and bisexual men. Drugs and Alcohol Today. https://doi.org/10.1108/DAT-12-2020-0083 


\section{Introduction}

'Chemsex' refers to the use of psychoactive drugs among gay and bisexual men (GBM) in sexualized settings. In recent years, the practice has emerged as an important public health concern. Engagement in chemsex is associated with negative physical and psychological health outcomes, such as infection with HIV and hepatitis C, as well as low self-esteem, depression and psychosis (Bohn et al., 2020; Pufall et al., 2018). In addition to public health research, there has been a drive to understand the psychological underpinnings of chemsex behavior, that is, why GBM do it, the physical and psychological needs that the practice may be fulfilling, and how to prevent it (or at least reduce harm) (e.g., Evans, 2019; Jaspal, 2020a). Research suggests that GBM may engage in the practice because it enhances sex, removes inhibitions, facilitates intimacy and provides a sense of escape (Weatherburn et al., 2017). A theoretical rationale has been provided for examining the relationship between chemsex behavior and identity processes (see Jaspal, 2018, 2020a), but research focusing specifically on the role of identity is scarce. Drawing on Identity Process Theory, this study focuses on GBM's motivations for engaging in chemsex and the functions that the practice performs for constructing a positive sense of self.

\section{Chemsex: Its definition and prevalence}

Chemsex commonly involves the use of mephedrone, crystal methamphetamine, $\gamma$ hydroxybutyrate (GHB), $\gamma$-butyrolactone (GBL), and crystallized methamphetamine, which are also colloquially referred to as 'chems.' Use of these substances in sexualized settings is intended to facilitate and enhance sex - in group or dyadic contexts - that may last for hours or days (Maxwell, Shahmanesh \& Gafos, 2019). In chemsex settings, substances may be used in combination with others, including alcohol, and some chemsex users also progress from initially ingesting substances to injecting them (often referred to as 'slamming'). Not all chemsex is necessarily problematic - some GBM engage in the practice transiently, employ risk reduction strategies and never feel the need to seek support for chemsex. However, many men do experience physical and/ or psychological adversity and are in need of support (Smith \& Tasker, 2017).

The practice was first observed in major cities in Western Europe and North America but is increasing globally. Stuart (2019) outlines the development of chemsex in the UK, noting its proliferation as technologies, such as the Internet and then geospatial gay social networking applications, have become available. He has argued that chemsex can be understood only in the social and cultural context of gay sexuality, which is characterized by long-standing stigma, trauma and a search for belonging. Thus, chemsex encompasses more than just taking drugs and having sex - rather, it represents the interdependence of the two activities.

It is difficult to estimate the prevalence of chemsex in GBM. Most previous studies have relied on convenience, rather than representative, samples and participants have often been recruited within sexual health clinic settings (introducing possible sampling bias). However, the available evidence suggests that a significant minority of GBM engage in chemsex (in its broadest sense). For instance, self-report survey data from 1484 GBM recruited from 20 sexual health clinics in the UK showed a 21.8 per cent prevalence of chemsex in the past 3 months (Sewell et al., 2017). Furthermore, GBM living with HIV are much more likely to report chemsex than those who are HIV-negative (Pufall et al., 2018).

\section{Motivations for chemsex}

Chemsex is by no means a uniform, homogeneous practice and men's motivations for doing it are similarly diverse. In their qualitative interview study of 30 chemsex users, Weatherburn et al. (2017) noted that chemsex could reportedly enhance one's sexual performance (through increased libido, self-confidence and stamina), make others seem more attractive, increase 
physical sensations and enhance feelings of intimacy and adventure. Overall, individuals may come to find sexual experiences during chemsex more pleasurable than in sober settings. The notion that chemsex facilitates disinhibition would appear to be consistent with the type of sexual activity that is often associated with the practice - chemsex participants are more likely to report engagement in esoteric, stigmatized sexual behaviors, such as fisting, which they might not ordinarily engage in when sober (Maxwell, Shahmanesh \& Gafos, 2019).

There is clearly also a strong psychological dimension to chemsex (Coston \& Rutter, 2020). Several studies have found that chemsex constitutes a form of escapism from the stressors that gay report in their everyday lives (van Hout et al., 2019; Weatherburn et al., 2017). These may include homonegativity, rejection and HIV stigma. In their qualitative study, Pollard, Nadarzynski and Llewellyn (2018) discuss the insidious effects of multi-pronged marginalization, namely homonegativity, an antithetical gay culture and adverse interpersonal experiences, noting that collectively they may culminate in chemsex. Furthermore, in their analysis of gay men's 'survival stories' of chemsex, Smith and Tasker (2017) noted that their participants' experiences of chemsex were rooted in their personal biographies as gay men facing thwarted feelings of belonging and acceptance. For them, chemsex may constitute a means of enhancing (and even 'regaining') their gay identity through self-exploration and selfdiscovery. It has been found that GBM living with HIV are more likely to engage in chemsex than their HIV-negative counterparts (Maxwell, Shahmanesh \& Gafos, 2019). By doing so, individuals may be coping with the stress and stigma of their HIV diagnosis (Jaspal \& Bayley, 2020). Consequently, for some GBM at least, chemsex appears to be fulfilling key psychological needs.

There is a growing empirical focus on pleasure in relation to chemsex and it is often argued that an exclusively risk- and harm-based approach to chemsex is limiting and misleading (Krysdale et al., 2020). In their focus group study of former and current chemsex participants in Montreal, Canada, Flores-Aranda et al. (2019) found that interviewees reported feeling more attractive to others and more confident with sexual partners and that they perceived a removal of barriers to achieving intimacy with others. Milhet et al. (2019) also focused on 'pleasure narratives' in the analysis of interview data from 33 chemsex users. They found that chemsex enabled individuals to develop and maintain feelings of interpersonal connectedness and that it facilitated self-expression and identity development by allowing participants 'to let themselves go'. However, there has been no examination of the potentially positive functions that chemsex may perform for identity processes. In order to understand the social psychological significance of chemsex for GBM who do it, it is valuable to view chemsex through the lens of identity.

\section{Identity processes}

Identity Process Theory (IPT) (Breakwell, 1986; Jaspal, 2018) posits that individuals strive to construct an identity characterized by satisfactory levels of self-esteem, self-efficacy, continuity and positive distinctiveness. These are referred to as identity principles. Identity is defined as the product of social and psychological processes and is constantly in flux - as the individual navigates the social world, they will assimilate-accommodate new elements into the identity structure and evaluate them. The evaluation of identity elements is a constant process, subject not only to individual beliefs and attitudes but also to broader social representations and ideologies that characterize their social context. For instance, as gay men initiate chemsex, they will need to take a stance on the practice, that is, to append meaning and value to it; they will need to assimilate it into the identity structure and view it as a part of who they are; and, in doing so, they may need to re-think other elements of their identity content (e.g., being a member of a friendship group that stigmatizes chemsex) so that it come to form part of it. 
A key tenet of IPT is that, when the identity principles are undermined or curtailed, the individual experiences identity threat which is harmful for psychological wellbeing. People engage in varied activity, operating at various levels of human functioning, in order to reduce or resolve threats to identity. At a psychological level, people may engage in deflection strategies, which reflect the refusal to acknowledge, accept or modify identity content due to a threat. For instance, individuals may fantasize in order to replace their threatening reality with a more psychologically acceptable one. At a collective level, individuals may engage in groups and social networks that provide increased feelings of self-esteem, self-efficacy and so on.

People may become accustomed to particular ways of coping due to previous life experiences, the opportunities available to them, and the social context which append value to some coping strategies and stigma to others (Jaspal, Lopes \& Wignall, 2020). Chemsex has been described in terms of 'escapism', that is, as a behavioral strategy that enables users to disengage from the psychological adversity that they face and to derive feelings of self-esteem and self-confidence (Weatherburn et al., 2017). Thus, chemsex may be conceptualized as an approach to coping with threats to identity. Through the lens of IPT, this study examines the role of chemsex as a possible coping resource.

\section{Participants}

\section{Method}

Using a snowball sampling strategy, 16 GBM were recruited primarily at four HIV/ sexual health charities located in London and in the East Midlands of England. Participants were invited to take part in an interview study concerning engagement in chemsex, focusing on their motivations for doing so and the impact of the practice on their identities. All 16 participants protected current engagement in chemsex. Participants were aged between 22 and 47 years. Three self-identified as bisexual and the rest as gay. Ten participants reported being HIVnegative and six as living with HIV. In terms of ethnicity, twelve individuals were White British and three were of Black Caribbean heritage and one was of Pakistani descent. Ten participants had university-level qualifications, and the remaining 6 had completed college education. Fourteen interviewees described themselves as being "completely out" as gay/bisexual and two as "closeted" to family and most friends. However, only six participants were "completely open" about their chemsex engagement and 10 participants generally concealed this information from others, including healthcare professionals. Five participants reported being in a romantic relationship and 11 as being single.

\section{Data generation and analysis}

The interviews were guided by a semi-structured interview schedule which consisted of several exploratory, open-ended questions focusing on self-description and identity, experiences of 'coming out' as gay/ bisexual, attitudes toward chemsex, motivations for engaging in chemsex, and the perceived functions of the practice for one's sense of identity. Interviews lasted between 60 and 80 minutes, and were digitally recorded and transcribed verbatim.

Qualitative thematic analysis, which has been described as 'a method for identifying, analyzing and reporting patters (themes) within data' (Braun \& Clarke, 2006, p. 78), was used to analyze the data. Thematic analysis enables the analyst to identify themes in the interviews that address the research questions. In this study, it was used in conjunction with tenets of IPT in order to shed light on the social psychological motivations of chemsex and its functions for identity processes.

Following the analytic steps for thematic analysis outlined in Jaspal (2020b), each of the transcripts was initially read and re-read and, during this process, preliminary analytic observations were noted. These initial codes mainly captured constructs relevant to the psychological aspects of chemsex. These initial codes were then collated into broader themes, 
which focused on the identity functions of chemsex. Finally, the themes were merged into three superordinate themes which reflected the overall analysis. They were ordered into a coherent narrative structure and specific interview extracts, which were considered illustrative of the themes, were included for presentation in the article.

\section{Identity threat and the allure of chemsex}

\section{Findings}

Interviewees unanimously reported long-term exposure to psychological stressors associated with their gay identity, such as homonegativity, rejection and victimization. In many cases, they described early childhood experiences of stigma which persisted in adulthood, albeit in different guises:

School was tough, you know. I was bullied every day and I was like really ashamed, sort of, to reach out because it was bullying because I'm gay or they called me a sissy... It made me ashamed, you know, about who I am and being gay and that... I think that [shame] has stayed with me even 'til this day. (Richard, 36)

Coming out to my parents was horrible. They accepted me in the end but the memorable bit that you always remember, that sticks with you, that was horrible. My mother cried in my face and even though they got to grips, came to terms with it, you just know it's something they wish you're not. I still think to myself 'she was never totally happy with this and isn 't'. (Mark, 33)

Although these adverse experiences were rooted in childhood, there was a sense that their adverse psychological effects were still felt in adulthood. For instance, Richard described the persistent feelings of shame in relation to his sexual orientation which persisted decades after his early experiences of bullying at school. Similarly, Mark recalled his negative experience of 'coming out' as gay to his mother, which generated the persistent belief that his mother was, and remained, unhappy about his sexual orientation. All of the interviewees described psychologically challenging experiences of this kind, which were unique to them as gay men struggling to construct and reconcile their sexual identity. Recalling these experiences was clearly threatening for self-esteem and distinctiveness. Participants generally described a decreased sense of self-worth because of their treatment by others and there was a pervasive sense that one had been 'excessively' and indeed negatively distinctive due to one's sexual orientation.

Individuals reported a sense of 'continuation' in their exposure to sexuality-specific stressors. While during childhood and early adulthood they had mainly faced homonegativity, in their present lives they reported facing stigma, judgment and rejection on the gay scene, that is, from other gay men. This appeared to 're-activate' threats to identity relating to their selfesteem and distinctiveness:

The gay scene is bad, wherever you go. In London, it's very judgmental because on the apps [gay social networking mobile applications] if you haven't got a six pack, you're fat. If you're not the alpha male, you're camp. It's hard to see how anyone has any ounce of self-value on the scene. It actually makes you actively like feel really unattractive and undesirable...There's none of that at chill-outs [chemsex gatherings] (Richard, 36)

I've stopped going on the scene because these fucking professional gays, they can be massively bitchy, just looking you up and down. It makes you feel like scum and you can't talk to anyone. Anyone that says 'gay community' needs a reality check. There is no gay community.. Who needs community when you've got chems? (Peter, 24) 
I don't do clubs. It ain't worth the hassle with the twats that go. I only do chill-outs, put it that way. Why would you put yourself through that shit? (Ian, 26)

Telling people I'm [HIV] positive is the hardest thing ever. The rejection, the hate you get it's horrible... When you're doing chems, no one's bothered about that, like it's not a barrier (Ian, 26)

In these extracts, interviewees are describing various specific stressors that they experience on the gay scene. Richard referred to body image concerns and the stigma of femininity (being perceived to be 'camp'). Peter described judgement and marginalization from 'professional gays' whom he perceived to be prototypical of contemporary gay male culture and emphatically challenged the notion of a 'gay community'. In a similar vein, Ian had reportedly given up on clubs, because of the negative interpersonal experiences that he had with other gay men in that context. He refused to put himself in a situation where he might face threats to identity. All six of the participants who were living with HIV reported exposure to HIV stigma, which constituted an additional psychological stressor which they encountered on the gay scene (see Jaspal, 2018). Yet, as Ian noted, chemsex offered temporary respite from stigma and exclusion. Indeed, most interviewees reported seeking solace on the chemsex scene, partly because of the acceptance and inclusion that they were afforded in this context vis-à-vis the gay scene (and indeed in sober settings). In fact, they actively contrasted their threatening experiences on the gay scene with the comparably accommodating context of chemsex.

\section{Deflection to cope with identity threat}

There was a general preference to engage in deflection strategies for coping with chronic threats to identity. For instance, some individuals suppressed memories of their adverse experiences, while others concealed their identity from others in order to shield themselves from stigma:

There's only so much you can take as a kid or even as a grown-up man so I just stopped thinking about the bullying... But it does come up now and then, not that I want it to. (Keiron, 47)

The gay bashing. I just zoned out and tried to put it down to 'they're just messing about, like a joke'...I was just not wanting to admit to myself that they think I'm gay because I didn't want to. I still don't like to admit it. (Kevin, 39)

Although some participants attempted to suppress memories of their adverse experiences, these memories continually gained access to consciousness, thereby demonstrating the limited efficacy of deflection as a coping strategy. Some interviewees described re-construing their experiences of bullying due to their sexual orientation, that is, they changed the meaning of these experiences to reduce their adverse impact on identity processes. Furthermore, Keith described still concealing his sexuality in some contexts in order to limit the risk of negative affect (e.g., shame) and identity threat:

I reckon I was kind of conditioned to hide it [being gay] if I could, I mean, I guess it was just the shame of it. (Keith, 39)

Chronic threats to identity shaped patterns of cognition, affect and behavior in relation to participants' sexual orientation. All of the interviewees acknowledged having experienced challenges in relation to 'coming out' and recounted experiences of disdain and hostility from significant others. Many resorted to negatively evaluating their sexual orientation, that is, 
internalized homonegativity (Berg, Munthe-Kaas \& Ross, 2016). This in turn led some to refrain from seeking social support and to individualize their coping strategies, often by deflecting threat:

I don't think I've ever recovered since those days because I do still feel low about it and there's stuff that bothers me that's from those days and the way I deal with stuff and even how I feel about me and being gay and that. It's a sort of trauma. I have to remind myself that I'm not 12 no more... I just don't find it easy to talk about it. I rather just run away from my problems. (Andrew, 23)

I've had body images issues all my life. You get more conscious of what you look like. Do I look too gay? Too fat? Do I look like a typical camp guy?...It feels like my own issues and I got to sort it myself but, yeah, I think it's from those dark days [of bullying] (Aaron, 27)

In reflecting on his identity, Andrew himself recognized remnants of his past experience and past coping styles in his current identity. Similarly, Aaron referred to his chronic self-esteem issues as being rooted in his childhood experiences of bullying but also that he now had a consequential proneness to self-doubt and self-questioning. Although interviewees attempted to break free from their deep-rooted psychological schemata, these experiences continued to mar both their self-image and their coping styles when facing re-emergent or novel threats to identity. The findings indicated that there was a habitual use of deflection strategies for coping with identity threat and that chemsex may be a key facilitator of this approach to coping.

\section{Chemsex as a gateway to deflection}

Engagement in chemsex appeared to constitute an approach to coping with identity threat and for enhancing feelings of self-esteem, positive distinctiveness and self-efficacy:

Doing chems, for me, it's been like an amazing experience, the ultimate high. You just lose all your barriers, all the things that hold you back and just let yourself go...I remember, the first time was awkward at first but someone gave me some $G$ and that was it, it was like I was let out and I was shocked at myself for doing stuff I would never do in the real world. It was like a liberation of the senses...It just makes you feel like you can do anything, liberated. (Philip, 41)

For the first time in my life, I actually felt desirable and I was doing stuff that I always wanted to, with guys that I'd never have the confidence to approach if I wasn't high and they weren't, so it was like entering a new world, a much better world than the one I was living in (Kevin, 39)

Sexual disinhibition was a pervasive theme and there was a strong sense among interviewees that chemsex enabled them to achieve things that might not ordinarily be possible. Like several others, Philip reported being relieved of the 'barriers' and 'things that hold you back', which engendered a sense of liberation. Similarly, upon initiating chemsex use, Kevin gained a greater sense of control and competence since he felt more confident about engaging in sexual practices with others than he did previously. In short, chemsex appeared to facilitate feelings of self-efficacy among users since they felt more empowered to achieve their (sexual) goals than when sober. Moreover, there was a sense that self-esteem was being enhanced - for Kevin, the chemsex scene constituted a 'much better world' because he felt desirable.

In contrast to mainstream society in which stressors were acutely felt (e.g., homonegativity), chemsex was construed as buffering their negative psychological effects: 
Being gay in our society isn't easy. Never has been. But when you do chems, it's like all your worries and your self-created barriers are just taken out the equation and that feels good (Andrew, 23)

I suppose it's like a safe space isn't it? Where you can just leave your skeletons out, I mean, like your own issues, you know, it's all in your head. You just feel good about yourself for once (Mark, 33)

In particular, there was a sense that chemsex safeguarded one's identity so that it was no longer imperiled by the 'self-created barriers' or 'skeletons' associated with everyday life as a gay man. Engagement in chemsex enabled some to re-construe their psychological challenges and their significance for identity. In the chemsex environment, they felt freed from these challenges and perceived an opportunity to derive feelings of self-esteem - for Mark at least, this opportunity was unprecedented and unparalleled. Individuals described two separate worlds - mainstream society in which they were exposed to sexuality-specific stressors and the chemsex environment in which these stressors simply disappeared. It was in the latter context that identity was anaesthetized from threat. Chemsex was clearly functioning as a generalized approach for coping with sexuality-related stressors.

Interviewees struggled to describe their psychological experiences during chemsex sessions but all converged in highlighting the euphoria they generated. Under close scrutiny, chemsex appeared to facilitate access to specific deflection strategies, which might not ordinarily be available to them in sober settings:

I'll go to a chill-out and it's like my dreams are coming true. The best way I can describe it is like an outer-body experience. I can't believe what I'm doing when I'm doing it. I'm loving it but it's like I'm watching myself and liking. I like that me. It's the chems I guess. (Peter, 24)

Doing chems is like a dream as in I'm having a really nice dream about yourself, totally different from when you wake up, so you don't want to wake up from because it feels good...it's happening and can see it but you're not like doing it there then. It's dream-like (Lewis, 23)

Chemsex appeared to enable some individuals to engage in the strategy of transient depersonalization, which has been defined as 'the fleeting momentary experience of feeling estranged from oneself' (Breakwell, 1986, p. 86). Consistent with previous research into chemsex (Weatherburn et al., 2017), Peter reported feeling sexually disinhibited and empowered to engage in activities that he would not ordinarily engage in. He described this as an 'outer-body experience' and himself rather like a spectator or onlooker observing his own behavior in chemsex sessions. This transient depersonalization allowed some to derive selfesteem - indeed, Peter noted liking the facet of himself that he observed, as a spectator, during chemsex sessions. Similarly, Lewis described his experience of transient depersonalization during chemsex using the analogy of having a pleasant dream which 'feels good'. He too alluded to the positive implications of his experience for self-esteem, which he contrasted with the reality of his decreased self-esteem outside of chemsex settings. Thus, chemsex served to alleviate a chronically threatened sense of self-esteem and enabled individuals to re-construe their reality in more psychologically acceptable ways:

I spent my whole life convincing myself about what I should be thinking and whatnot...Chemsex is a whole other level...It's like when I'm there that all didn't happen and I'm something I know 
I'm not, like one time I felt like a pornstar [laughs] I'm just whatever I want to be and that's the main thing when I'm there (Aaron, 27)

I'll tell you what I do...when I'm there at that moment in time, it just feels like this is forever. I'm not exactly at a chill-out thinking 'oh yeah, I better make the most of it'. It actually feels like all my past is erased and this is my whole world and that feels amazing. The best feeling ever. The pain comes afterwards (Andrew, 23)

In contrast to the coercive social norms about sexuality and the heteronormativity that interviewees reported facing during their lives, chemsex provided access to an alternative psychological reality which was deemed to be more satisfying for identity processes. It enabled them to re-construct their identity more favorably. The 'fantasy world' afforded to them during chemsex sessions was described in various ways. Aaron noted his negative body image and low self-esteem, which he contrasted with his fantasy self-construal as a pornstar during chemsex sessions. These fantasies were sustained by a temporary erasure of the past. Consistent with the observation that chemsex constitutes a form of escapism (Jaspal \& Bayley, 2020), individuals sustained their more psychologically satisfying version of reality by compartmentalizing past and present. The past was temporarily forgotten and a transient rupture in continuity was necessary to enable chemsex to generate feelings of self-esteem. A key observation across the entire participant sample was that the feelings of self-esteem and self-efficacy provided by chemsex were transient - as Andrew stated, 'the pain comes afterwards'.

\section{Identity threat and coping}

\section{Discussion}

Some GBM are exposed to long-standing stressors, namely homonegativity, stigma on the gay scene and, in some cases, HIV stigma. These specific stressors have been examined extensively elsewhere (e.g., Berg, Munthe-Kaas \& Ross, 2016; Jaspal \& Bayley, 2020). The aim of this study was not to provide a re-examination of these stressors but to understand their potential effects on identity and the social psychological backdrop against which GBM may be engaging in chemsex. In particular, the principles of self-esteem and distinctiveness appeared to be chronically susceptible to threat. Interviewees reported feeling less able to derive a positive self-conception and excessively and negatively distinctive (due, for instance, to being gay, HIV-positive). Chemsex appeared to address these threats to identity and was also conducive to an increased sense of self-efficacy. When engaging in chemsex, participants may feel shielded from stressors that compromise a positive self-conception and, given the increased sense of disinhibition reported in this context, they may be empowered to engage in practices and to achieve things that are not deemed possible in sober settings.

Consistent with IPT, GBM who face identity threat attempt to cope. The findings demonstrate an over-reliance on deflection strategies for coping with existent and emergent threats to identity. Interviewees reported having suppressed memories of adversity, concealing them from others and re-construing their meanings. Many reported having been socialized in a heteronormative and, in many cases homonegative, social context which precluded the derivation of social support. Many described negative reactions to their attempts to seek support, such as when coming out to significant others. Many lamented the lack of an effective gay community from which social support could be derived. Therefore, one had been virtually primed to believe that (effective) interpersonal and group-based coping strategies, such as selfdisclosure and social support, were unavailable. Jaspal, Lopes and Wignall (2020) have described various coping styles, that is, clusters of coping strategies that individuals habitually use in response to identity threat. Choice of coping strategy is determined, at least in part, by 
past experience. The GBM interviewed in this study expressed a long-standing preference for deflection, that is, an escape from psychological stress.

\section{Escapism in chemsex clarified}

Chemsex constitutes a form of escapism (Jaspal \& Bayley, 2020). This study shows that engagement in chemsex constituted a 'gateway' to additional, more elaborate strategies for deflecting threats to identity.

First, there was a focus in the findings on transient depersonalization, which enabled individuals to 'stand outside of themselves and observe themselves in a detached, disconnected way' (Breakwell, 1986, p. 86). Some of the interviewees struggled to articulate this psychological experience, generally referring to themselves as spectators during chemsex, observing themselves enact behaviors that they would not adopt in sober settings. The strategy was deployed to protect identity from threat since it essentially disconnected the individual from the stressors that perhaps prompted them to seek solace on the chemsex scene in the first place. The past, consisting of these stressors, and the present characterized by one's positive chemsex experience were essentially compartmentalized - the past was simply unable to compromise the present. By engaging in transient depersonalization, individuals were able to focus, as spectators, on the identity-enhancing aspects of their chemsex experience.

Second, chemsex enabled individuals to fantasize in order to enhance identity processes. More specifically, this strategy allowed them to replace their threatening reality (associated with the 'outer world') with a more psychologically satisfying reality. They were able to construct a more positive identity, characterized by self-esteem and self-efficacy, on the basis of an imagined reality. Some could focus on a facet of identity that they desired but could not attain in sober settings. Others lost all concept of time believing their sensory experiences during chemsex to be ever-lasting. Chemsex gave this reality credence as it was essentially 'cordoned off' from the real world which might question it.

\section{Conclusion}

This study demonstrates the importance of contextualizing chemsex behavior in the identity narratives of GBM. Indeed, in reflecting on their chemsex behavior, most interviewees returned to childhood experiences whose psychological effects could be felt in the present. GBM are habitually exposed to multiple social psychological stressors, which can increase their risk of chronic, unresolved threats to identity. Interviewees reported a preference for deflection strategies for coping with threat. This was attributed to a long-standing coping style rooted in childhood and sustained by subsequent adverse experiences in adulthood. For GBM who struggle to derive social and interpersonal support, deflection may be deemed preferable to running the risk of further rejection.

Chemsex appears to constitute a gateway to additional, more elaborate deflection strategies, namely transient depersonalization, compartmentalization and fantasy. While intended to protect identity from threat, these deflection strategies are unlikely to be effective in the long term. They may escalate into delusions and hallucinations that extend beyond the confines of the chemsex environment. Indeed, severe psychopathology of this kind has been reported in relation to chemsex (Bohn et al., 2020). Understanding the identity functions performed by chemsex is important. This will be a crucial step toward understanding why GBM do it. It will be vital for designing interventions to prevent chemsex in GBM vulnerable to identity threat and for supporting those who are asking for help.

\section{Declaration of conflicting interests}

The author declared no potential conflicts of interest with respect to the research, authorship, and/or publication of this article. 


\section{Funding}

The author received no funding for this study.

\section{References}

Berg, R.C., Munthe-Kaas, H.M., \& Ross, M.W. (2016). Internalized homonegativity: A systematic mapping review of empirical research. Journal of Homosexuality, 63(4), 541-558. https://doi.org/10.1080/00918369.2015.1083788.

Bohn, A., Sander, D., Köhler, T., Hees, N., Oswald, F., Scherbaum, N., Deimel, D., \& Schecke, H. (2020). Chemsex and mental health of men who have sex with men in Germany. Frontiers in Psychiatry, 11, 542301. https://doi.org/10.3389/fpsyt.2020.542301

Breakwell, G.M. (1986). Coping with threatened identities. London: Methuen.

Evans, K. (2019). The psychological roots of chemsex and how understanding the full picture can help us create meaningful support. Drugs and Alcohol Today, 19(1), 36-41. https://doi.org/10.1108/DAT-10-2018-0062

Flores-Aranda, J., Goyette, M., Aubut, V., Blanchette, M., \& Pronovost, F. (2019). Let's talk about chemsex and pleasure: the missing link in chemsex services. Drugs and Alcohol Today, 19(3), 189-196. https://doi.org/10.1108/DAT-10-2018-0045

Jaspal, R. (2018). Enhancing Sexual Health, Self-Identity and Wellbeing among Men Who Have Sex With Men: A Guide for Practitioners. London: Jessica Kingsley Publishers

Jaspal, R. (2020a). Chemsex among men who have sex with men: A social psychological perspective. In M. Croston \& S. Rutter (eds.), Psychological Perspectives in HIV care (pp.124143). London: Routledge. https://doi.org/10.4324/9781315211404-7

Jaspal, R. (2020b). Content analysis, thematic analysis and discourse analysis. In G.M. Breakwell, D.B. Wright \& J. Barnett (eds.), Research Methods in Psychology (5th Edition) (pp. 285-312). London: Sage.

Jaspal, R., \& Bayley, J. (2020). HIV and Gay Men: Clinical, Social and Psychological Aspects. London: Palgrave. https://doi.org/10.1007/978-981-15-7226-5

Jaspal, R., Lopes, B., \& Wignall, L. (2020). The Coping with Identity Threat Scale: Development and validation in a university student sample. Identity: An International Journal of Theory and Research, 20(4), 225-238. https://doi.org/10.1080/15283488.2020.1808469

Maxwell, S., Shahmanesh, M., \& Gafos, M. (2019). Chemsex behaviours among men who have sex with men: A systematic review of the literature. International Journal on Drug Policy, 63, 74-89. https://doi.org/10.1016/j.drugpo.2018.11.014

Milhet, M., Shah, J., Madesclaire, T., \& Gaissad, L. (2019). Chemsex experiences: Narratives of pleasure. Drugs and Alcohol Today, 19(1), 11-22. https://doi.org/10.1108/DAT-09-2018$\underline{0043}$ 
Pollard, A., Nadarzynski, T., \& Llewellyn, C. (2018). Syndemics of stigma, minority-stress, maladaptive coping, risk environments and littoral spaces among men who have sex with men using chemsex. Culture, Health \& Sexuality, 20(4), 411-427. https://doi.org/10.1080/13691058.2017.1350751

Pufall, E.L., Kall, M., Shahmanesh, M., Nardone, A., Gilson, R., Delpech, V., Ward, H., \& Positive Voices Study Group (2018). Sexualized drug use ('chemsex') and high-risk sexual behaviours in HIV-positive men who have sex with men. HIV Medicine, 19(4), 261-270. https://doi.org/10.1111/hiv.12574

Sewell, J., Miltz, A., Lampe, F.C., Cambiano, V., Speakman, A., Phillips, A.N., ... the AURAH Study Group. (2017). Poly drug use, chemsex drug use, and associations with sexual risk behaviour in HIV-negative men who have sex with men attending sexual health clinics. International Journal of Drug Policy, 43, 33-43. https://doi.org/10.1016/j.drugpo.2017.01.001

Smith, V. \& Tasker, F. (2017) Gay men's Chemsex survival stories. Sexual Health, 15(2), 116122. https://doi.org/10.1071/SH17122

Stuart, D. (2019). Chemsex: Origins of the word, a history of the phenomenon and a respect to the culture. Drugs and Alcohol Today, 19(1), 3-10. https://doi.org/10.1108/DAT-10-2018-0058

Van Hout, M. C., Crowley, D., O'Dea, S., \& Clarke, S. (2019). Chasing the rainbow: Pleasure, sex-based sociality and consumerism in navigating and exiting the Irish Chemsex scene.

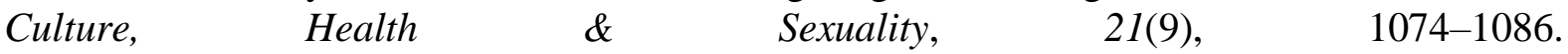
https://doi.org/10.1080/13691058.2018.1529336

Weatherburn, P., Hickson, F., Reid, D., Torres-Rueda, S., \& Bourne, A. (2017). Motivations and values associated with combining sex and illicit drugs ('chemsex') among gay men in South London: findings from a qualitative study. Sexually Transmitted Infections, 93(3), 203206. https://doi.org/10.1136/sextrans-2016-052695 